\title{
ENDOMORPHISMS AND AUTOMORPHISMS FROM SUBFACTORS ILLUSTRATING NON-COMMUTATIVE ENTROPY
}

\author{
ANNE LOUISE SVENDSEN
}

\begin{abstract}
We present a series of examples of endomorphisms and automorphisms arising from subfactors, which illustrate some of the recent theorems in non-commutative entropy theory. Moreover it is shown that for these examples the Connes-Størmer entropy of the automorphism is maximal and coincides with the topological entropy. This follows from the theorems our examples illustrate, and is also showed directly.
\end{abstract}

\section{Introduction}

In the papers [15] and [16] Wenzl constructed new examples of subfactors by using representations of Hecke algebras and representations of BirmanWenzl-Murakami algebras, which in particular are representations of the Braid group. This construction was generalized by Erlijman [5] who considers general representations of the Braid group subject to a certain set of conditions. Erlijman then constructs the subfactor generated by the representation of a two-sided sequence of Braid group generators. We consider these subfactors along with the subfactors generated by a one-sided sequence of Braid group generators according to Wenzl's construction. These examples have a natural homomorphism, which shifts the Braid group generators. When we consider the one-sided sequence we get an endomorphism, and when we consider the two-sided sequence we get an automorphism. The main part of this paper is devoted to showing how the above mentioned examples illustrate some of the recent theorems in the theory of non-commutative entropy, namely the Variational principle; see [9], the McMillan theorem; see [10], and the theorem relating the entropy to the index, see [12]. Thus we will work in the interplay between subfactors and entropy. Both the index of subfactors; see [8], the Connes-Størmer entropy; see [4], the entropy in $C^{*}$-algebras; see [3], and Voiculescu's approximation entropy; see [14], come into play. For a survey of the results on the different entropies; see [13]. In addition to the results by

Received June 16, 2003; in revised form November 29, 2004. 
Wenzl and Erlijman, we will also be using other known results, in particular by Choda. Some of these results are only refered to, but others are included in order to make the exposition more coherent.

The paper is organized as follows. In section 2 we present the above mentioned theorems and show how these results can be used to prove that the entropy coincide with the topological entropy under certain special conditions. In section 3 we state the conditions Erlijman requires the representations of the Braid group to satisfy, and show how properties of the endomorphism/automorphism follow from these. We show that the endomorphisms illustrate the theorem relating the entropy to the index, whereas the automorphisms illustrate the Variational principle and the McMillan theorem. Moreover it follows that the automorphisms in the examples have maximal entropy, which equals the topological entropy. In section 4 we show for completeness that this result can also be showed directly for both the automorphism and the endomorphism using only that we have periodic inclusions and a mean generator.

\section{Theorems about entropy}

We consider $C^{*}$-dynamical systems $(A, \tau, \alpha)$, where $\mathrm{A}$ is a unital $C^{*}$-algebra, $\tau$ is a trace on $A$, and $\alpha$ is a $\tau$-preserving automorphism of $A$. Throughout the paper we will implicitly use the fact that when computing the entropy we can move back and forth between a $C^{*}$-algebra $A$ with a trace $\tau$ and an automorphism $\alpha$, and the von Neumann algebra $M=\pi_{\tau}(A)^{\prime \prime}$ (the GNSrepresentation) with $\tilde{\alpha}$ the extension of $\alpha$; see [13]. In order to formulate the Variational principle we recall the following definition.

DEFINITION 2.1. A $C^{*}$-dynamical system $(A, \tau, \alpha)$ is called asymptotically abelian with locality, if there exists a dense $\alpha$-invariant subalgebra $B$ of $A$ such that for all pairs $a, b \in B$ the algebra $C^{*}(a, b)$ is finite dimensional, and there is a $p=p(a, b) \in \mathrm{N}$ such that $\left[\alpha^{j}(a), b\right]=0$ whenever $|j| \geq p$.

THEOREM 2.2 (Variational principle [9]). Let $(A, \tau, \alpha)$ be a unital separable $C^{*}$-dynamical system which is asymptotically abelian with locality. Let $H \in$ $A_{\text {sa }}$, where $A_{\text {sa }}$ denotes the self-adjoint elements of $A$. Then the pressure $P_{\alpha}$ satisfies

$$
P_{\alpha}(H)=\sup _{\phi}\left(h_{\phi}(\alpha)-\phi(H)\right)
$$

where the sup is taken over all $\alpha$-invariant states. In particular the topological entropy satisfies

$$
\operatorname{ht}(\alpha)=\sup _{\phi} h_{\phi}(\alpha) .
$$

For asymptotically abelian algebras with locality, the size of the minimal projections can be estimated by the McMillan theorem. 
Theorem 2.3 (McMillan [10]). Let $(A, \tau, \alpha)$ be a unital separable $C^{*}$ dynamical system which is asymptotically abelian with locality. Suppose the trace $\tau$ is an extremal $\alpha$-invariant state, and that the entropy $H(\alpha)$ is finite. Moreover suppose that $N$ is a finite dimensional subalgebra of $A$, which is a mean generator, i.e.

$$
H(\alpha)=\lim _{n \rightarrow \infty} \frac{1}{n} H\left(N_{n}\right)<\infty,
$$

where $N_{n}=\bigvee_{i=0}^{n-1} \alpha^{i}(N)$. Then given $\epsilon>0$ there exists an $N \in \mathrm{N}$ such that for all $n \geq N$ there exists a central projection $z_{n} \in N_{n}$ such that $\tau\left(z_{n}\right)<\epsilon$ and

$$
\exp (-n(H(\alpha)+\epsilon))<\tau(p)<\exp (-n(H(\alpha)-\epsilon))
$$

for all minimal projections $p$ in the algebra $N_{n}\left(1-z_{n}\right)$.

A general definition of a mean generator, which we will use from now on, is the following. If $\left(A_{n}\right)$ is an increasing sequence of finite dimensional algebras which generate $A$, and $\alpha$ is an automorphism or an endomorphism of $A$, then $\left(A_{n}\right)$ is called a mean generator for the entropy of $\alpha$ if $H(\alpha)=\lim _{n} \frac{1}{n} H\left(A_{n}\right)$. The definition of a mean generator in the theorem above is a special case of this when the algebra has locality. Namely let $A_{n}=\vee_{i=0}^{n-1} \alpha^{i}(N)=N_{n}$, then $\left(A_{n}\right)$ is an increasing sequence of finite dimensional subalgebras of $A$, since $\mathrm{N}$ is finite dimensional and we have locality. Moreover $\lim _{n} \frac{1}{n} H\left(A_{n}\right)=$ $\lim _{n} \frac{1}{n} H\left(N_{n}\right)=H(\alpha)$. On the other hand if $\alpha\left(A_{n}\right) \subset A_{n+1}$, we can fix $k$ and let $N=A_{k}$. It then follows that $N_{n}=\vee_{i=0}^{n-1} \alpha^{i}(N) \subset A_{k+n-1}$, hence $\lim _{n} \frac{1}{n} H\left(N_{n}\right) \leq \lim _{n} \frac{1}{n} H\left(A_{k+n-1}\right)=H(\alpha)$.

By using the McMillan theorem in conjunction with the Variational principle we get sufficient conditions for the Connes-Størmer entropy to be maximal and coincide with the topological entropy.

Corollary 2.4. Let $(A, \tau, \alpha)$ be a unital separable $C^{*}$-dynamical system which is asymptotically abelian with locality, and where $\tau$ is extremal among the $\alpha$-invariant states. Suppose that $A$ is an AF-algebra with a generating sequence $\left(A_{n}\right)$, which is a mean generator for the entropy, and such that $\alpha\left(A_{n}\right) \subset A_{n+1}$. Let $t_{n}, d_{n}$ denote the trace vector and the dimension vector, respectively, of the state $\tau$ restricted to the algebra $A_{n}$. Suppose that $\lim _{n \rightarrow \infty} t_{n}(i) d_{n}(i) \geq \delta>0$ for all $i$. Then

$$
H(\alpha)=\sup _{\phi} h_{\phi}(\alpha)=\operatorname{ht}(\alpha),
$$

where the sup is taken over all $\alpha$-invariant states. 
Proof. Fix an $\alpha$-invariant state $\phi$. In particular this could be $\tau$. By the Kolmogorov-Sinai theorem for the entropy of $C^{*}$-algebras; see [13], we have $h_{\phi}(\alpha)=\lim _{n \rightarrow \infty} h_{\phi, \alpha}\left(A_{n}\right)$. But since $\alpha\left(A_{n}\right) \subset A_{n+1}$ this implies that

$$
\begin{aligned}
h_{\phi}(\alpha) & =\lim _{n} h_{\phi, \alpha}\left(A_{n}\right)=\lim _{n} \lim _{k} \frac{1}{k} H_{\phi}\left(A_{n}, \alpha\left(A_{n}\right), \ldots, \alpha^{k-1}\left(A_{n}\right)\right) \\
& \leq \lim _{n} \limsup _{k} \frac{1}{k} H_{\phi}\left(A_{n+k-1}\right) \leq \limsup _{k} \frac{1}{k} H_{\phi}\left(A_{k}\right) .
\end{aligned}
$$

(The argument above is for instance used in [1] for the Connes-Størmer entropy.) So it is enough to show that $H(\alpha) \geq \lim \sup _{k} \frac{1}{k} H_{\phi}\left(A_{k}\right)$. Let $\epsilon<\delta$ be given. By the McMillan theorem (theorem 2.3) there is an $N \in \mathrm{N}$ such that for all $n \geq N$ there exists a central projection $z_{n} \in A_{n}$ such that $\tau\left(z_{n}\right)<\epsilon$ and

$$
\exp (-n(H(\alpha)+\epsilon))<\tau(p)<\exp (-n(H(\alpha)-\epsilon))
$$

for all minimal projections $p$ in the algebra $A_{n}\left(1-z_{n}\right)$. But since we chose $\epsilon<$ $\delta$ we must have $z_{n}=0$ by the assumption, since both the trace vector and the dimension vector have strictly positive entries, and $\tau\left(z_{n}\right)=\sum_{i \in I} t_{n}(i) d_{n}(i)$ for some subset $I$ of $\mathrm{N}$. Thus we have (*) for all minimal projections $p_{n} \in A_{n}$ for all $n \geq N$, hence $\lim _{n \rightarrow \infty} \frac{-1}{n} \log \tau\left(p_{n}\right)=H(\alpha)$. By concavity of the function $\eta(t)=-t \log t$, it therefore follows that the entropy from the trace is the maximal entropy. Finally we get

$$
H(\alpha)=\sup _{\phi} h_{\phi}(\alpha)=\operatorname{ht}(\alpha)
$$

by the Variational principle (theorem 2.2).

We recall the following definition of periodicity; see [15].

Definition 2.5. Let $A_{1} \subset A_{2} \subset \cdots$ be an increasing sequence of finite dimensional $C^{*}$-algebras. Such a sequence is called periodic (of period $k$ ) if there is an $N \in \mathrm{N}$ and a $k \in \mathrm{N}$, such that for all $n \geq N$ the inclusion $A_{n+i k} \subset A_{n+(i+1) k}$ is given by a primitive matrix $G_{n}$ for all $i \in \mathrm{N}$, (recall that a matrix $G$ is primitive if $G$ is a square matrix and there exists an $l \in \mathrm{N}$ such that $G^{l}$ has positive entries), and $k$ is the smallest value such that this holds.

REMARK 2.6. Let $G$ be a primitive $r \times r$ matrix. Then the largest eigenvalue (the Perron-Frobenius value) $\beta$ is strictly positive. Let $\xi$ be the normalized eigenvector to $\beta$. We will use the following facts from Perron-Frobenius theory [6].

- Up to multiplication by a positive scalar the eigenvector $\xi$ is unique and has strictly positive entries. 
- $\bigcap_{l \in \mathrm{N}} G^{l}\left(\mathrm{R}_{+}^{r}\right)=\mathrm{R}_{+} \xi$.

- $\lim _{l \rightarrow \infty} \beta^{-l} G^{l} w=(w, \xi) \xi$ for any $r$-dimensional vector $w$, where $(\cdot, \cdot)$ denotes the inner product of the two vectors.

The requirement in the above corollary about the size of the central projections is satisfied when the generating sequence is periodic.

Corollary 2.7. Let A be an AF-algebra generated by the increasing sequence $\left(A_{n}\right)$, which is periodic. Let $t_{n}, d_{n}$ denote the trace vector and the dimension vector of the state $\tau$ restricted to the algebra $A_{n}$, respectively. Then there is a $\delta$ such that

$$
\lim _{n \rightarrow \infty} t_{n}(i) d_{n}(i) \geq \delta>0 \quad \text { for all } i .
$$

Proof. Suppose the period of the sequence $\left(A_{n}\right)$ is $k$. Fix $m \in \mathbf{N}$ such that the inclusion $A_{m+i k} \subset A_{m+(i+1) k}$ is given by the matrix $G$ for all $i \in \mathbf{N} \cup\{0\}$, which we from now on will write as $A_{m+i k} \underset{G}{\subset} A_{m+(i+1) k}$. Then $d_{m+i k}=$ $G^{i} d_{m}$. Denote the Perron-Frobenius value by $\beta$, the unique normalized PerronFrobenius eigenvector to $\beta$ by $\xi$, and suppose the primitive inclusion matrix $G$ is an $r \times r$ matrix. For all $i$ we have $t_{m+i k}=G^{l} t_{m+(i+l) k}$ for all $l \in \mathrm{N}$. Thus for each $i$ we have $t_{m+i k} \in \cap_{l \in \mathbf{N}} G^{l}\left(\mathrm{R}_{+}^{r}\right)$, hence in particular $t_{m+i k}=\lambda_{i} \xi$ for some $\lambda_{i}>0$, i.e.

$$
t_{m}=G^{l} t_{m+l k}=G^{l} \lambda_{l} \xi=\beta^{l} \lambda_{l} \xi=\beta^{l} t_{m+l k} .
$$

Moreover by Perron-Frobenius theory $\beta^{-l} G^{l} d_{m} \rightarrow\left(d_{m}, \xi\right) \xi$ for $l \rightarrow \infty$. Thus we get for each $j \in \mathbf{N}$

$$
\begin{aligned}
\lim _{n} d_{n}(j) t_{n}(j) & =\lim _{l} d_{m+l k}(j) t_{m+l k}(j)=\lim _{l} G^{l} d_{m}(j) \beta^{-l} t_{m}(j) \\
& =\lim _{l}\left(\beta^{-l} G^{l} d_{m}(j)\right) t_{m}(j)=\left(d_{m}, \xi\right) \xi(j) \lambda_{0} \xi(j) \\
& =\left(d_{m}, t_{m}\right)(\xi(j))^{2} \lambda_{0}=(\xi(j))^{2} \lambda_{0}>0,
\end{aligned}
$$

where we used that $\left(d_{m}, t_{m}\right)=\sum_{i=1}^{r} d_{m}(j) t_{m}(j)=\tau(1)=1$, and noted the independence of the fixed $m \in \mathbf{N}$. Since $\xi$ is a finite dimensional vector we can choose $0<\delta<\min _{j}\left(\xi(j)^{2}\right) \lambda_{0}$.

In the cases where the trace is extremal the two corollaries above prove the following.

Corollary 2.8. Let $(A, \tau, \alpha)$ be a unital separable $C^{*}$-dynamical system which is asymptotically abelian with locality, and where $\tau$ is extremal among the $\alpha$-invariant states. Suppose that $A$ is an AF-algebra with a generating 
sequence $\left(A_{n}\right)$, which is both periodic and a mean generator for the entropy. Then

$$
H(\alpha)=\sup _{\phi} h_{\phi}(\alpha)=\operatorname{ht}(\alpha)
$$

where the sup is taken over all $\alpha$-invariant states.

\section{The examples from subfactors}

Recall that the Braid group $B_{n}$ on $n$ strands is the free group in the generators $\sigma_{1}, \ldots, \sigma_{n-1}$ subject to the relations

- $\sigma_{i+1} \sigma_{i} \sigma_{i+1}=\sigma_{i} \sigma_{i+1} \sigma_{i}$ for $1 \leq i \leq n-2$.

- $\sigma_{i} \sigma_{j}=\sigma_{j} \sigma_{i}$ whenever $|i-j| \geq 2$.

In the paper [5] Erlijman works with representations $\rho$ of the algebra $C B_{\infty}$ satisfying the following properties with $C_{n}=\rho\left(\mathrm{C} B_{n}\right)$ :

(1) For each $n \in \mathrm{N}: \rho\left(\mathrm{C} B_{n}\right) \cong \bigoplus_{\lambda \in \Lambda_{n}} M_{a_{\lambda}}$ for some finite index set $\Lambda_{n}$.

(2) $g_{i}:=\rho\left(\sigma_{i}\right)$ is unitary for all $i$.

(3) The sequence of finite dimensional $C^{*}$-algebras $\left(C_{n}\right)$ is periodic.

(4) Any $x \in C_{n+1}$ is of the form $x=a g_{n}^{ \pm 1} b+c$, where $a, b, c \in C_{n}$.

(5) The unique trace $\tau$ on $C=\overline{\bigcup_{n} C_{n}}$ is a Markov trace, i.e. $\tau\left(g_{n}^{ \pm 1} x\right)=$ $\eta \tau(x)$ for all $n \in \mathrm{N}$ and all $x \in C_{n}$. In particular this implies that $\tau(x y)=\tau(x) \tau(y)$ when $x$ and $y$ are in subalgebras generated by disjoint subsets of the generators $g_{i}^{ \pm 1}$.

(6) For all $k \in \mathrm{N}$ there exists a projection $p \in C_{k}$ such that for all $n \in \mathrm{N}$ we have

$$
p C_{n+k} p \cong p C_{k+1, n+k} \cong C_{k+1, n+k} \cong C_{n},
$$

where $C_{s, t}$ is the subalgebra generated by $g_{s}, \ldots, g_{t-1}$.

Examples of representations with the above properties arise from representations of Hecke algebras and Birman-Murakami-Wenzl algebras as mentioned previously.

In the rest of this paper $\rho$ will be a representation satisfying the above properties, and $\alpha$ will be the shift of the generators $g_{i}$, i.e. $\alpha\left(g_{i}\right)=g_{i+1}$ for all $i$.

REMARK 3.1. It can be shown from property (5) (also denoted the strongly clustering property) that the unique trace is extremal among the set of $\alpha$ invariant states on $C$. For a proof of this see [11].

REMARK 3.2. In the rest of this paper we will several times use the Kolomogorov-Sinai theorem for endomorphisms instead of for automorphisms. For 
the Connes-Størmer entropy the proof of this is a straightforward adaption of the proof of the Kolomogorov-Sinai theorem for automorphisms by using that $H(\alpha(A) \mid \alpha(B)) \leq H(A \mid B)$ when $\alpha$ is an endomorphism; see [1]. For the entropy of $C^{*}$-algebras it follows directly from the usual theorem. This is also the case for the topological entropy, but with the following minor change in the assumptions. We consider finite subsets $\left(\omega_{j}\right)$ of the nuclear $C^{*}$-algebra $A$ with $\omega_{1} \subset \omega_{2} \subset \cdots$. For automorphisms the requirement is that the linear span of $\cup_{j \in \mathbf{N}, k \in Z} \alpha^{k}\left(\omega_{j}\right)$ is dense in $A$. For endomorphisms we make the obvious adjustment and require that the linear span of $\cup_{j \in \mathbb{N}, k \in \mathbb{N}} \alpha^{k}\left(\omega_{j}\right)$ is dense in $A$, then the same proof works.

The examples of endomorphisms and automorphisms that we will consider are of the "shift"-type. The next well-known lemma shows that they have a mean generator.

LEMMA 3.3. Let $A$ be an AF-algebra generated by the increasing sequence $\left(A_{n}\right)$. Let $\tau$ be a trace on $A$ and let $\alpha$ be a trace-preserving endomorphism or automorphism of $A$. Suppose that

(1) $A_{n} \vee \alpha\left(A_{n}\right) \vee \alpha^{2}\left(A_{n}\right) \vee \cdots \vee \alpha^{j}\left(A_{n}\right) \cong B_{n+j}$, where $B_{n+j}$ is a subalgebra of $A_{n+j}$ for all $n, j \in \mathrm{N}$.

(2) $\left[A_{n}, \alpha^{i n}\left(A_{n}\right)\right]=0$ for all $i \geq 1$.

(3) $\tau\left(\alpha^{i n}(x) y\right)=\tau(x) \tau(y)$ for all $x, y \in A_{n}$ for any $n \in \mathrm{N}$, for all $i \geq 1$.

Then the sequence $\left(A_{n}\right)$ is a mean generator for the entropy, i.e. $H(\alpha)=$ $\lim _{n} \frac{1}{n} H\left(A_{n}\right)$.

Proof. Since $\left(A_{n}\right)$ is an increasing sequence and $A=\overline{\cup_{n \in \mathrm{N}} A_{n}}$, we have $H(\alpha)=\lim _{n \rightarrow \infty} H\left(A_{n}, \alpha\right)$ by the Kolomogorov-Sinai theorem for endomorphisms or automorphisms; see [13]. Now on the one hand we have

$$
\begin{aligned}
H(\alpha) & =\lim _{n} H\left(A_{n}, \alpha\right)=\lim _{n} \lim _{k} \frac{1}{k} H\left(A_{n}, \alpha\left(A_{n}\right), \ldots, \alpha^{k-1}\left(A_{n}\right)\right) \\
& \leq \limsup _{k} \frac{1}{k} H\left(A_{k}\right)
\end{aligned}
$$

by using (1) as in corollary 2.4 . On the other hand we have

$$
n H(\alpha)=H\left(\alpha^{n}\right) \geq \lim _{k} \frac{1}{k} H\left(A_{n}, \alpha^{n}\left(A_{n}\right), \alpha^{2 n}\left(A_{n}\right), \ldots, \alpha^{(k-1) n}\left(A_{n}\right)\right) .
$$

By (2) the algebras $A_{n}$ and $\alpha^{i n}\left(A_{n}\right)$ commute for all $i \geq 1$, hence it follows that

$$
H\left(A_{n}, \alpha^{n}\left(A_{n}\right), \ldots, \alpha^{(k-1) n}\left(A_{n}\right)\right)=H\left(\bigvee_{i=0}^{k-1} \alpha^{i n}\left(A_{n}\right)\right)
$$


Moreover by (3) we have $\tau(x y)=\tau(x) \tau(y)$ for $x \in \alpha^{i n}\left(A_{n}\right)$ and $y \in \alpha^{j n}\left(A_{n}\right)$ with $i \neq j$, hence

$$
H\left(\bigvee_{i=0}^{k-1} \alpha^{i n}\left(A_{n}\right)\right)=\sum_{i=0}^{k-1} H\left(\alpha^{i n}\left(A_{n}\right)\right)=\sum_{i=0}^{k-1} H\left(A_{n}\right)=k H\left(A_{n}\right),
$$

by [13]. Therefore

$$
n H(\alpha) \geq \lim _{k} H\left(A_{n}\right)=H\left(A_{n}\right),
$$

i.e. $H(\alpha) \geq \lim _{n} \frac{1}{n} H\left(A_{n}\right)$, thus all in all we have $H(\alpha)=\lim _{n} \frac{1}{n} H\left(A_{n}\right)$.

\subsection{The endomorphism from the one-sided sequence}

Consider the sequence of algebras $\left(C_{n}\right)$ defined above, i.e. $C_{n}=\left\langle g_{1}, \ldots g_{n-1}\right\rangle$. Define the endomorphism $\alpha$ on the generators by $\alpha\left(g_{i}\right)=g_{i+1}$ for $i \geq 1$. Then $\alpha$ is trace-invariant hence extends by continuity to the von Neumann algebra $C={\overline{\cup_{n \in \mathrm{N}} C_{n}}}^{w}$, which is the hyperfinite $\mathrm{II}_{1}$ factor due to the uniqueness of the trace. This sequence is periodic (property 3)). Let $D_{n}=\left\langle g_{2}, \ldots g_{n-1}\right\rangle$ for $n \in \mathrm{N}$ and let $D={\overline{\cup_{n \in \mathrm{N}} D_{n}}}^{w}$. Then $D$ is a subfactor of $C$. It is shown in [15] by use of Wenzl's dimension estimate that the subfactor is irreducible.

For the relation between the entropy of an endomorphism and the index of the subfactor generated by the endomorphism, we have the following theorem.

THEOREM 3.4 ([12]). Let $R$ be the hyperfinite $\mathrm{II}_{1}$ factor with trace $\tau$ and a $\tau$-preserving endomorphism $\alpha$. Let $\left(A_{n}\right)$ be an increasing sequence of finite dimensional algebras generating $R, A_{n}=\bigoplus_{l \in K_{n}} M_{l}^{n}$, where $M_{l}^{n}$ is a factor of type $m_{l}^{n}$. Let $a_{k l}^{n}$ denote the multiplicity of $\alpha\left(M_{k}^{n-1}\right)$ in $M_{l}^{n}$. Suppose there is a $q \in \mathrm{R}$ such that for all $k, l, n$ we have $a_{k l}^{n} \leq q m_{k}^{n-1}$. Moreover suppose that $\alpha\left(A_{n}\right) \subset A_{n+1}$ for all $n \in \mathrm{N}$,

$$
\lim _{n \rightarrow \infty} \frac{1}{n} H\left(A_{n}\right)=H(\alpha)<\infty,
$$

and that

$$
\begin{array}{ccc}
A_{n+1} & \subset & A_{n+2}, \tau \\
\cup & & \cup \\
\alpha\left(A_{n}\right) & \subset & \alpha\left(A_{n+1}\right)
\end{array}
$$

is a commuting square for all $n \in \mathrm{N}$. Let $Z\left(A_{n}\right)$ denote the center of the algebra $A_{n}$. Then

(1) $\lim _{n \rightarrow \infty} \frac{1}{n} H\left(Z\left(A_{n}\right)\right)$ exists.

(2) $R \cap \alpha(R)^{\prime}$ is atomic with minimal projections $f_{k}, \sum_{k} f_{k}=1$. 
(3) $H(\alpha)=H\left(R \cap \alpha(R)^{\prime}\right)+\frac{1}{2} \tau\left(f_{k}\right) \log \left[R_{f_{k}}: \alpha(R)_{f_{k}}\right]+\frac{1}{2} \lim _{n \rightarrow \infty} \frac{1}{n} H\left(Z\left(A_{n}\right)\right)$.

The theorem above differs from the one in [12] by the extra condition of the limited growth of the multiplicities $\left(a_{k l}^{n} \leq q m_{k}^{n-1}\right)$, since we can not make the proof of the theorem work without this. On the other hand we have not been able to show that this extra condition is necessary.

Let us show that the above theorem is illustrated by the series of examples coming from shifts on the one-sided sequence. We start with a straightforward lemma; see [5], [15], and [16].

Lemma 3.5. Let $C_{1} \subset C_{2} \subset \cdots \subset C$ be given as above. Then the following properties hold:

(1) $C_{n} \vee \alpha\left(C_{n}\right) \vee \alpha^{2}\left(C_{n}\right) \vee \cdots \vee \alpha^{j}\left(C_{n}\right)=C_{n+j}$ for all $n, j \in \mathrm{N}$.

(2) $\left[C_{n}, \alpha^{i n}\left(C_{n}\right)\right]=0$ for all $i \geq 1$.

(3) $\tau\left(\alpha^{i n}(x) y\right)=\tau(x) \tau(y)$ for all $x, y \in C_{n}$ for any $n \in \mathrm{N}$, for all $i \geq 1$.

Lemma 3.6. The increasing sequence of finite dimensional algebras $\left(C_{n}\right)$ defined at the beginning of this section satisfies the hypotheses of theorem 3.4.

Proof. Let $n \in \mathrm{N}$. Since $C_{n}=\left\langle g_{1}, \ldots g_{n-1}\right)$, we have $\alpha\left(C_{n}\right)=$ $\left\langle g_{2}, \ldots, g_{n}\right\rangle \subset C_{n+1}$. By lemmas 3.3 and 3.5 the sequence $\left(C_{n}\right)$ is a mean generator. We need to see that

$$
\begin{aligned}
& C_{n+1} \subset C_{n+2}, \tau \quad\left\langle g_{1}, \ldots, g_{n}\right\rangle \subset\left\langle g_{1}, \ldots, g_{n+1}\right\rangle, \tau \\
& \alpha\left(C_{n}\right) \subset \alpha\left(C_{n+1}\right) \quad\left\langle g_{2}, \ldots, g_{n}\right\rangle \subset\left\langle g_{2}, \ldots, g_{n+1}\right\rangle
\end{aligned}
$$

is a commuting square. This is proved in [15], but we include the argument here for the sake of completeness. Let $y \in C_{n+1}, x \in \alpha\left(C_{n+1}\right)$. Then by property 4) we can assume without loss of generality that $x$ has the form $x=\alpha\left(a g_{n}^{ \pm 1} b\right)$, where $a, b \in C_{n}$. So we get that

$$
\begin{aligned}
\tau\left(E_{\alpha\left(C_{n+1}\right)}(y) x\right) & =\tau\left(E_{\alpha\left(C_{n+1}\right)}(y x)\right)=\tau(y x)=\tau\left(y \alpha\left(a g_{n}^{ \pm 1} b\right)\right) \\
& =\tau\left(y \alpha(a) g_{n+1}^{ \pm 1} \alpha(b)\right)=\eta \tau(y \alpha(a) \alpha(b)) \\
& =\eta \tau\left(E_{\alpha\left(C_{n}\right)}(y) \alpha(a b)\right)=\tau\left(E_{\alpha\left(C_{n}\right)}(y) x\right) .
\end{aligned}
$$

Thus $E_{\alpha\left(C_{n+1}\right)}(y)=E_{\alpha\left(C_{n}\right)}(y) \in \alpha\left(C_{n}\right)$, hence we have a commuting square. Finally, with the notation of theorem 3.4 , we see that there exists a $q$ such that $a_{k l}^{n} \leq q m_{k}^{n-1}$ for all $k, l, n$, since the sequence $\left(C_{n}\right)$ is periodic and $\alpha\left(C_{n}\right) \subset$ $C_{n+1}$. 
From the lemma above and theorem 3.4, we get the following conclusion, which is also proved in [1] and [2] by different methods without appealing to theorem 3.4.

Corollary 3.7. For the sequence $\left(C_{n}\right)$ we get that

$$
H(\alpha)=\frac{1}{2} \log [C: D] .
$$

Proof. By the above lemma the hypotheses of theorem 3.4 are satisfied. Thus we get that

$$
H(\alpha)=H\left(C \cap \alpha(C)^{\prime}\right)+\frac{1}{2} \tau\left(f_{k}\right) \log \left[C_{f_{k}}: \alpha(C)_{f_{k}}\right]+\frac{1}{2} \lim _{n \rightarrow \infty} \frac{1}{n} H\left(Z\left(C_{n}\right)\right),
$$

where $f_{k}$ are minimal projections in $C \cap \alpha(C)^{\prime}$ with $\sum_{k} f_{k}=1$. But $C \cap$ $\alpha(C)^{\prime}=C \cap D^{\prime}=\mathrm{C}$, since the subfactor is irreducible, hence $H\left(C \cap \alpha(C)^{\prime}\right)=$ $H(\mathrm{C})=0$. Since the sequence $\left(\operatorname{dim}\left(Z\left(C_{n}\right)\right)\right)$ is bounded because the inclusion $\left(C_{n}\right)$ is periodic, this implies that $\lim _{n \rightarrow \infty} \frac{1}{n} H\left(Z\left(C_{n}\right)\right)=0$, thus the result follows.

Note that by theorem 3.4 (and the above corollary) we have two different ways of computing the entropy or the index. This is also described in section 6.2 of [12], where theorem 3.4 is applied to similar examples where the algebra is generated by a series of Jones projections.

\subsection{The automorphism from the two-sided sequence}

The representation $\rho$ is extended to the two-sided infinite Braid group. In order to get a mean generator for the entropy we define the increasing sequence of $C^{*}$-algebras $\left(A_{n}\right)$ as follows.

DeFINITION 3.8. Let

$$
\begin{aligned}
A_{2 n} & =\left\langle g_{-(n-1)}, \ldots, g_{-1}, g_{0}, g_{1}, \ldots g_{n-1}\right\rangle, \\
A_{2 n+1} & =\left\langle g_{-(n-1)}, \ldots g_{-1}, g_{0}, g_{1}, \ldots, g_{n}\right\rangle
\end{aligned}
$$

for $n \in \mathrm{N}$.

Let $A=\overline{\bigcup_{n \in \mathrm{N}} A_{n}}$ and let $\tau$ be the unique trace on $A$, a property which $A$ inherits from $\overline{\bigcup_{n} C_{n}}$. The sequence $\left(A_{n}\right)$ is periodic, since the sequence $\left(C_{n}\right)$ is periodic and $\left(A_{2 n} \subset A_{2 n+1}\right) \cong\left(C_{2 n} \subset C_{2 n+1}\right)$ by the automorphism which shifts the generators up by $n$. Let $\alpha$ be the automorphism of $A$, which shifts the generators, i.e. $\alpha\left(g_{i}\right)=g_{i+1}$ for all $i \in \mathrm{Z}$ extended to the full algebra $A$. Since $\tau$ satisfies property 5), $\alpha$ is trace-preserving. 
The following lemma follows from lemma 3.5 using that $A_{n+j} \cong$ $\left\langle g_{1}, \ldots, g_{n+j}\right\rangle$.

Lemma 3.9. Let $A_{1} \subset A_{2} \subset \cdots \subset A$ be given as above. Then the following properties hold:

(1) $A_{n} \vee \alpha\left(A_{n}\right) \vee \alpha^{2}\left(A_{n}\right) \vee \cdots \vee \alpha^{j}\left(A_{n}\right) \cong B_{n+j}$, where $B_{n+j}$ is a subalgebra of $A_{n+j}$ for all $n, j \in \mathrm{N}$.

(2) $\left[A_{n}, \alpha^{i n}\left(A_{n}\right)\right]=0$ for all $i \in \mathbf{Z} \backslash\{0\}$.

(3) $\tau\left(\alpha^{i n}(x) y\right)=\tau(x) \tau(y)$ for all $x, y \in A_{n}$ for any $n \in \mathrm{N}$, for all $i \in$ $\mathbf{Z} \backslash\{0\}$.

Corollary 3.10. For the $C^{*}$-dynamical system $(A, \tau, \alpha)$ defined above we have

$$
H(\alpha)=\sup _{\phi} h_{\phi}(\alpha)=\mathrm{ht}(\alpha) .
$$

Proof. The trace $\tau$ is unique and the sequence $\left(A_{n}\right)$ is periodic, so by lemma 3.9 the requirements for lemma 3.3 are satisfied, hence the sequence $\left(A_{n}\right)$ is a mean generator for the entropy. Moreover $(A, \tau, \alpha)$ is a unital, separable, $C^{*}$-dynamic system, which is asymptotically abelian with locality. Indeed in definition 2.1 we can use $B=\bigcup_{n \in \mathrm{N}} A_{n}$. Let $a, b \in A$, i.e. there are $n, m \in \mathbf{N}$ such that $a \in A_{n}$ and $b \in B_{m}$. Let $l=\max \{n, m\}$. Then we have $C^{*}(a, b) \subset A_{l}$, which is finite dimensional. Moreover let $p=p(a, b)=n m$, then $\left.\left[\alpha^{j}(a), b\right)\right]=0$ whenever $|j| \geq p$ by lemma 3.9. Hence the system is asymptotically abelian with locality. By remark 3.1 the trace is extremal among the $\alpha$-invariant states, so since $\left(A_{n}\right)$ is a mean generator for the entropy with respect to $\tau$, it follows from corollary 2.8 that $H(\alpha)=\sup _{\phi} h_{\phi}(\alpha)=\operatorname{ht}(\alpha)$.

\section{Algebras generated by periodic inclusions}

For algebras generated by a periodic sequence of finite dimensional algebras, the entropy of an endomorphism can be computed using the following theorem due to Choda; see [1]. We repeat here the proof for the sake of completeness, since we will use an argument of the same type in the next theorem. Also a similar argument is already used in corollary 2.7.

TheOREM 4.1 ([1]). Let A be an AF-algebra generated by the increasing sequence of finite dimensional $C^{*}$-algebras $\left(A_{n}\right)$, i.e. $A_{1} \subset A_{2} \subset \cdots \subset$ $\overline{\bigcup_{n \in \mathrm{N}} A_{n}}=A$. Let $\tau$ be a trace on $A$ and let $\alpha$ be a trace-preserving endomorphism or automorphism of $A$. Suppose that the sequence $\left(A_{n}\right)$ is periodic and a mean generator for the entropy, i.e.

$$
H(\alpha)=\lim _{n} \frac{1}{n} H\left(A_{n}\right) .
$$


Then

$$
H(\alpha)=\frac{1}{k} \log \beta,
$$

where $k$ is the period and $\beta$ is the Perron-Frobenius value of the primitive inclusion matrix $G$.

Proof. Fix $m \in \mathrm{N}$ such that $A_{m+i k} \underset{G}{\subset} A_{m+(i+1) k}$ for all $i \in \mathrm{N} \cup\{0\}$. Suppose the primitive inclusion matrix $G$ is an $r \times r$ matrix. Denote the Perron-Frobenius value by $\beta$, and denote the unique normalized Perron-Frobenius eigenvector to $\beta$ by $\xi$. Finally denote the trace vector and dimension vector of the trace $\tau$ restricted to the algebra $A_{n}$ by $t_{n}$ and $d_{n}$, respectively. As in the proof of corollary 2.7 we have $t_{m}=G^{l} t_{m+l k}=\beta^{l} t_{m+l k}$. Thus

$$
\begin{aligned}
H\left(A_{m+l k}\right) & =-\sum_{i=1}^{r} d_{m+l k}(i) t_{m+l k}(i) \log \left(t_{m+l k}(i)\right) \\
& =-\sum_{i=1}^{r} d_{m+l k}(i) t_{m+l k}(i) \log \left(\beta^{-l} t_{m}(i)\right) \\
& =\sum_{i=1}^{r} d_{m+l k}(i) t_{m+l k}(i)(l \log \beta)-\sum_{i=1}^{r} d_{m+l k}(i) t_{m+l k}(i)\left(\log t_{m}(i)\right) \\
& \leq l \log \beta-\sum_{i=1}^{r}\left(\log t_{m}(i)\right),
\end{aligned}
$$

since $\sum_{i=1}^{r} d_{m+l k}(i) t_{m+l k}(i)=1$ is a sum of positive terms. Since the last term does not depend on $l$ we get that

$$
\lim _{n} \frac{1}{n} H\left(A_{n}\right)=\lim _{l} \frac{H\left(A_{m+l k}\right)}{m+l k}=\lim _{l} \frac{l}{m+l k} \log \beta=\frac{1}{k} \log \beta,
$$

thus all in all $H(\alpha)=\frac{1}{k} \log \beta$, which does not depend on $m$.

By using Choda's theorem we get the following result.

TheOREM 4.2. Let $A$ be an AF-algebra generated by the increasing sequence $\left(A_{n}\right)$. Let $\tau$ be a trace on $A$ and let $\alpha$ be a trace-preserving endomorphism or automorphism of $A$. Suppose that the sequence $\left(A_{n}\right)$ is periodic and a mean generator for the entropy, i.e.

$$
H(\alpha)=\lim _{n} \frac{1}{n} H\left(A_{n}\right),
$$


and suppose that $\alpha\left(A_{n}\right) \subset A_{n+1}$. Then

$$
H(\alpha)=\sup _{\phi} h_{\phi}(\alpha)=\operatorname{ht}(\alpha),
$$

where the sup is taken over all $\alpha$-invariant states.

Proof. We use the same notation as in the proof of theorem 4.1 and go through the case where $\alpha$ is an endomorphism. When $\alpha$ is an automorphism the proof is adjusted in the obvious way. By using the KolmogorovSinai theorem for endomorphisms of $C^{*}$-algebras, it is enough to show that $\lim \sup _{n} \frac{1}{n} H_{\phi}\left(A_{n}\right) \leq H(\alpha)$ for any $\alpha$-invariant state $\phi$ according to the proof of corollary 2.4. Let us show that we get a similar result for the topological entropy. Let $\mathscr{C} \mathscr{P} \mathscr{A}(A)$ denote the triples $(\phi, \psi, B)$, where $B$ is a finite dimensional $C^{*}$-algebra and $\phi: A \rightarrow B, \psi: B \rightarrow A$ are unital completely positive maps. Then the completely positive $\delta$-rank is defined by

$\operatorname{rcp}(\omega ; \delta)$

$=\inf \{\operatorname{rank} B \mid(\phi, \psi, B) \in \mathscr{C P} \mathscr{A}(A),\|(\psi \circ \phi)(a)-a\|<\delta$ for all $a \in \omega\}$,

for $\omega$ any finite subset of $A$.

We define the sequence of elements $\left(\omega_{n}\right)$ recursively. Let $\omega_{1}$ be an orthonormal basis for the algebra $A_{1}$. Then $\omega_{1}$ is a finite subset of $A$, since $A_{1}$ is finite dimensional. Extend $\omega_{1}$ to an orthonormal basis $\omega_{2}$ of $A_{2}$, extend this to an orthonormal basis $\omega_{3}$ of $A_{3}$ etc. This way we get an increasing sequence $\left(\omega_{n}\right)$ of finite elements of $A$. Moreover for each $i$ we have $\omega_{i} \subset A_{i}$. Since $\cup_{n} A_{n}$ is dense in $A$ we also have that the linear span of $\cup_{j \in \mathbb{N}, k \in \mathbb{N}} \alpha^{k}\left(\omega_{j}\right)$ is dense in $A$, since already the linear span of $\cup_{j \in \mathbb{N}} \omega_{j}$ is dense. Hence we can use the Kolmogorov-Sinai theorem for the topological entropy of the endomorphism to get that

$$
\mathrm{ht}(\alpha)=\sup _{j} \sup _{\delta>0} \lim \sup _{n} \frac{1}{n} \log \operatorname{rcp}\left(\cup_{i=0}^{n-1} \alpha^{i}\left(\omega_{j}\right) ; \delta\right) .
$$

But $\omega_{i} \subset A_{i}$, thus $\cup_{i=0}^{n-1} \alpha^{i}\left(\omega_{j}\right) \subset \cup_{i=0}^{n-1} \alpha^{i}\left(A_{j}\right) \subset A_{j+n-1}$. This implies that

$$
\operatorname{ht}(\alpha) \leq \sup _{j} \sup _{\delta>0} \limsup _{n} \frac{1}{n} \log \operatorname{rank}\left(A_{j+n-1}\right) \leq \limsup _{n} \frac{1}{n} \log \operatorname{rank}\left(A_{n}\right) .
$$

So it is enough to show that $\lim \sup _{n} \frac{1}{n} \log \operatorname{rank}\left(A_{n}\right) \leq H(\alpha)$. Let $\delta_{n}$ be the rank of the algebra $A_{n}$, i.e. $\delta_{n}=\sum_{i=1}^{r} d_{n}(i)$. By concavity of the function $\eta(t)=-t \log t$ we have $h_{\phi}\left(A_{n}\right) \leq \log \delta_{n}$. But since $d_{m+l k}=G^{l} d_{m}=$ 
$\beta^{l}\left(\beta^{-l} G^{l} d_{m}\right)$ we have

$$
\begin{aligned}
\log \left(\delta_{m+l k}\right) & =\log \left(\sum_{i=1}^{r} d_{m+l k}(i)\right)=\log \left(\sum_{i=1}^{r} \beta^{l}\left(\beta^{-l} G^{l} d_{m}(i)\right)\right) \\
& =l \log \beta+\log \left(\sum_{i=1}^{r} \beta^{-l} G^{l} d_{m}(i)\right) .
\end{aligned}
$$

By Perron-Frobenius theory $\beta^{-l} G^{l} d_{m} \rightarrow\left(d_{m}, \xi\right) \xi$ for $l \rightarrow \infty$, so

$$
\log \left(\delta_{m+l k}\right) \rightarrow l \log \beta+\log \left(\sum_{i=1}^{r}\left(d_{m}, \xi\right) \xi(i)\right)
$$

for $l \rightarrow \infty$. Hence it follows that

$$
\begin{aligned}
\lim _{n} \frac{1}{n} \log \left(\delta_{n}\right) & =\lim _{l} \frac{1}{m+l k} \log \left(\delta_{m+l k}\right)=\lim _{l} \frac{1}{m+l k}(l \log \beta) \\
& =\frac{1}{k} \log \beta=H(\alpha)
\end{aligned}
$$

by theorem 4.1. Thus the entropy from the trace is maximal and equals the topological entropy, since we always have the inequality $H(\alpha) \leq \mathrm{ht}(\alpha)$ [13].

By using the above theorem and the previous results it follows directly that the entropy for our series of examples (for both the automorphisms and the endomorphisms) is maximal and coincides with the topological entropy, since we have periodic inclusions and a mean generator.

Acknowledgment. The author would like to thank Professors Erling Størmer and Sergey Neshveyev, both University of Oslo, Norway, for many fruitful conversations.

\section{REFERENCES}

1. Choda, M., Entropy for *-endomorphisms and relative entropy for subalgebras, J. Operator Theory 25 (1991), 125-140.

2. Choda, M., Entropy for canonical shift, Trans. Amer. Math. Soc. 334 (1992), 827-849.

3. Connes, A., Narnhofer, H., and Thirring, W., Dynamical entropy of $C^{*}$-algebras and von Neumann algebras, Comm. Math. Phys. 112 (1987), 691-719.

4. Connes, A. and Størmer, E., Entropy for automorphisms of $I_{1}$ von Neumann algebras, Acta Math. 134 (1975), 289-306.

5. Erlijman, J., New subfactors from braid group representations, Trans. Amer. Math. Soc. 350 (1998), 185-211.

6. Gantmacher, F. R., Theory of Matrices, Vol. II, Chelsea Publications (1959). 
7. Hiai, F., Entropy for canonical shifts and strong amenability, Internat. J. Math. 6 (1995), 381-396.

8. Jones, V. F. R., Index for subfactors, Invent. Math. 72 (1983), 1-25.

9. Neshveyev, S., and Størmer, E., The variational principle for a class of asymptotically abelian $C^{*}$-algebras, Comm. Math. Phys. 215 (2000), 177-196.

10. Neshveyev, S., and Størmer, E., The McMillan theorem for a class of asymptotically abelian $C^{*}$-algebras, Ergodic Theory Dynam. Systems 22 (2002), 889-897.

11. Pedersen, G. K., $C^{*}$-Algebras and their Automorphism Groups, Academic Press Inc. (1979).

12. Størmer, E., Entropy of endomorphisms and relative entropy in finite von Neumann algebras, J. Funct. Anal. 171 (2000), 34-52.

13. Størmer, E., A survey of noncommutative dynamical entropy, Classification of nuclear $C^{*}$ algebras, Entropy in operator algebras, Encyclopaedia Math. Sci. 126 (2002), Springer, Berlin, 147-198.

14. Voiculescu, D., Dynamical approximation entropies and topological entropy in operator algebras, Comm. Math. Phys. 170 (1995), 249-281

15. Wenzl, H., Hecke algebras of type $A_{n}$ and subfactors, Invent. Math. 92 (1988), 349-383.

16. Wenzl, H., Quantum groups and subfactors of type B, C, and D, Comm. Math. Phys. 133 (1990), 383-432.

DEPARTMENT OF MATHEMATICS

UNIVERSITY OF OSLO

P.O. BOX 1053, BLINDERN

N-0316 OSLO

NORWAY

E-mail: annelsv@math.uio.no
CURRENT ADDRESS:

DEPARTMENT OF MATHEMATICS

UNIVERSITY OF COPENHAGEN

UNIVERSITETSPARKEN 5

DK-2100 COPENHAGEN $\varnothing$

DENMARK

E-mail: svendsen@math.ku.dk 